\title{
Manejo de residuos sólidos generados en el mercado central del municipio de Chiquimula
}

\section{Management of solid waste generated in the central market of the municipality of Chiquimula}

\begin{abstract}
Como citar el artículo
Casasola Garza, A. K. . Manejo de residuos sólidos generados en el mercado central del municipio de Chiquimula. Revista Naturaleza, Sociedad Y Ambiente, 8(1). DOI: https://doi.org/10.37533/cunsurori.v8i1.63
\end{abstract}

\section{Andrea Karina Casasola Garza}

Ministerio de Agricultura, Ganadería y Alimentación -MAGA

Recibido: 19 de abril de 2021 / Aceptado: 28 de junio de 2021

Disponible en internet el 10 de octubre de 2021

*Autor para correspondencia, correo electrónico: casasolaa@15gmail.com

\begin{abstract}
Resumen
El artículo hace referencia a la necesidad de contribuir con el manejo adecuado de los residuos sólidos que se generan actualmente en la plaza del mercado central del municipio de Chiquimula. El mercado central es uno de los comercios más importantes en el departamento y como cualquier otra actividad, el mercado contribuye a la generación de residuos. El manejo inadecuado de los residuos sólidos es uno de los principales problemas respecto al tema ambiental dado que no se cuentan con las medidas necesarias para mitigar los daños que afectan al bienestar de las personas. Sin duda alguna, las plazas de mercado presentan un carácter más definido aún en cuanto a la composición de los residuos, ya que ahí se concentran en su mayoría las ventas de verduras y frutas lo que implica que gran parte de los residuos sean de origen orgánico. Desde este contexto, la investigación se realizó con el objetivo de caracterizar los residuos que ahí se generan para la identificación de alternativas de manejo de los mismos.
\end{abstract}

Palabras clave: Residuos sólidos, caracterización, manejo de residuos, compostaje, residuos orgánicos.

\section{Abstract}

The article refers to the need to contribute to the adequate management of solid waste that is currently generated in the central market square of the municipality of Chiquimula. The central market is one of the most important businesses in the department and as such contributes to the generation of waste. The uneducated management of solid waste is one of the main problems regarding the environmental issue since there are no necessary measures to mitigate the damages that affect the well-being of people. Without a doubt, the market places are even more defined in terms of the composition of the waste, since the majority of sales of vegetables and fruits are concentrated there, which implies that a large part of the waste is of organic origin. . From this context, the research was carried out with the aim of characterizing the wastes generated there for the identification of their management alternatives..

Keywords: Solid waste, characterization, waste management, composting, organic waste 


\section{Introducción}

"Uno de los principales problemas ambientales en el territorio nacional es la generación de desechos sin tratamiento adecuado" (URL, 2009), el cual afecta la calidad de vida de las personas y el entorno natural. En el mercado central de Chiquimula se genera gran cantidad de residuos, la cual no tiene un manejo adecuado que busque de alguna manera aprovechar el alto potencial que tienen y que a su vez ayude a reducir el problema que producen. Debido a la importancia que tiene el manejo adecuado de los residuos sólidos en la actualidad, se hace necesario desarrollar estudios relativos al mismo, que permitan identificar alternativas para su gestión integral .

Cabe entonces indicar que el proyecto de investigación "Manejo de los residuos sólidos generados en la plaza del mercado central del municipio de Chiquimula, departamento de Chiquimula" tiene como objetivo general contribuir con el adecuado manejo de los residuos sólidos, mediante la identificación de alternativas que permitan hacer un adecuado manejo de los mismos.

En este artículo se presentan los resultados obtenidos de la caracterización en la cual fue necesaria la toma de muestras de los diferentes sectores identificados dentro del mercado central de Chiquimula. A través del análisis de las muestras se obtuvo información sobre la generación, composición y densidad de los residuos sólidos generados en la plaza del mercado central del municipio de Chiquimula durante la semana de monitoreo. Como primer paso se determinó la generación semanal de residuos obteniendo una producción de 30,717.91 lb. Seguidamente se determinó la composición física de los residuos sólidos a través del método del cuarteo obte- niendo los siguientes resultados: $74.94 \%$ de residuos sólidos, seguidamente con el $12.58 \%$ el plástico (todo tipo de plástico), papel y cartón con el $8.19 \%$, papel higiénico con el $1.99 \%$, vidrio con el $1.88 \%$, metal con el $0.24 \%$ y la clasificación de otros con el $0.22 \%$ del peso total. Como último paso de la caracterización también se determinó la densidad obteniendo densidades durante la semana de muestreó de $220.03 \mathrm{~kg} / \mathrm{m}^{3}$ a $237.71 \mathrm{~kg} / \mathrm{m}^{3}$.

Así mismo, se identificaron alternativas a través de las cuales se pretende manejar y disponer los residuos sólidos generados en la plaza del mercado central del municipio de Chiquimula, que contribuyan a la disminución de la contaminación y a la generación de ingresos económicos.

\section{Marco teórico}

\subsection{Residuos sólidos}

La Organización de las Naciones Unidas para el Desarrollo Industrial define residuo como "todo lo que es generado como producto de una actividad, ya sea por la acción directa del hombre o por la actividad de otros organismos vivos, formándose una masa heterogénea que, en muchos casos, es difícil de reincorporar a los ciclos naturales" (Férnandez y Sánchez, 2007).

Se puede considerar que los residuos sólidos son generados como resultado de las actividades que realiza la población para su subsistencia y para la obtención de insumos en los diferentes sectores productivos como son el comercio, la industria, el sector agropecuario y el de servicios (SEDESOL, 2005). Estos residuos sólidos son susceptibles o no de aprovechamiento 0 transformación para darle otra utilidad o uso directo. No tienen 
ningún valor económico para el usuario, pero sí un valor comercial para su recuperación e incorporación al ciclo de vida de la materia (Zamora, 2013).

\subsection{Generación de residuos y desechos sóli- dos en Guatemala}

El incremento en el consumo de productos procesados ha elevado la tasa de generación de desechos por habitante diaria. En Guatemala la generación per cápita de residuos y desechos sólidos comunes es de 0.519 kilogramos por habitante al día; en donde la composición de los residuos sólidos valorizables se estima en $53 \%$ de materia orgánica; $9 \%$ plásticos; $6 \%$ papel y cartón; $2 \%$ vidrio y $1 \%$ latas (MARN, 2018), el problema radica en que cada vez hay más productos que están fabricados para un solo uso, como lo expresa Echarri (1998:387) "se usan las cosas y se desechan en grandes cantidades, sin que haya conciencia clara, en muchos casos, de que luego algo hay que hacer con todos estos residuos".

Sin embargo, "muchos residuos se pueden reciclar si se dispone de las tecnologías adecuadas y el proceso es económicamente rentable" (Echarri, 1998), precisamente una buena gestión de los residuos busca no perder el valor económico utilizándolos como materia prima de nuevos productos.

\subsection{Residuos sólidos y su relación con la salud y el ambiente}

El problema de los desechos ha evolucionado junto con la urbanización, el crecimiento económico y la industrialización, "el deficiente manejo de los residuos sólidos contamina todos los factores ambientales (suelos, aire, aguas, paisaje, etc.) y, por consiguiente, afecta la salud de la población en su conjun- to" (Ayala, 2010). La búsqueda de una solución a un manejo adecuado de los residuos ha sido difícil, al punto que hoy, ni el marco legal, ni las políticas impulsadas por la mayoría de los gobiernos han dado un resultado satisfactorio (Aguilar e Iza, 2009).

En definitiva, el manejo de los residuos tiene una estrecha relación con la salud de la población y el medio ambiente. De acuerdo con Contreras (2008), se han presentado tres situaciones principales, la primera referida a la transmisión de enfermedades tanto por agentes patógenos transferidos por los residuos como por vectores, moscas, ratas, cucarachas que se alimentan y reproducen en los residuos y transmiten enfermedades bacteriales y parasitarias; en segundo lugar, el riesgo de lesiones e infecciones ocasionados por los objetos punzo penetrantes que se encuentran en los residuos, esta condición pone en riesgo la salud de las personas que recuperan materiales en los vertederos; y en tercer lugar, la contaminación producida por la quema de residuos, la cual afecta el sistema respiratorio de las personas.

\subsection{Factores que influyen en la generación y tipo de residuos sólidos}

La composición de residuos sólidos varía de acuerdo al nivel de desarrollo del país, nivel socioeconómico de la población, características climáticas, costumbres, hábitos de consumo, actividades económicas, poder adquisitivo de la población, incluso dentro de la misma ciudad, la composición puede diferir de acuerdo a los distintos estratos

socioeconómicos y a las diferencias entre las estaciones del año (OPS, 2005). 


\subsection{Gestión integral de los residuos sólidos}

De acuerdo con Zamora (2013), la gestión de los residuos inicia con el proceso de recolección, el transporte, tratamiento, reciclado y eliminación de los materiales de desecho, dicha gestión busca reducir sus efectos sobre la salud y el ambiente. La gestión de los desechos es también llevada a cabo para recuperar los propios recursos de dichos residuos.

Por lo tanto, la gestión integral de los residuos es aquel conjunto de acciones normativas, operativas, financieras, de planeación, administrativas, sociales, educativas, de monitoreo, supervisión y evaluación, para el manejo de residuos, desde su generación hasta la disposición final (ciclo de vida del residuo), a fin de lograr beneficios ambientales, la optimización económica de su manejo y su aceptación social, respondiendo a las necesidades y circunstancias de cada localidad o región (Aguilar e Iza, 2009).

Por su parte Alvarado (2010), establece que las prioridades en la gestión de los residuos sólidos se concretan de la siguiente manera:

- Prevención y minimización;

- Reutilización (especialmente para envases);

- Reciclado (papel, vidrio, plásticos, etc.);

- Valorización de la materia orgánica;

- Valorización energética; y

- Eliminación de vertederos, cumpliendo los requisitos técnicos establecidos.

La preferencia en el manejo de los residuos, es hacia la minimización de los residuos a depositar en los vertederos, "otorgando una alta prioridad a la recogida selectiva y al reciclado de diversos tipos de residuos como son: los papeles, vidrios, plásticos, escombros, materia orgánica, etc." (Férnandez y Sánchez, 2007).

\subsection{Clasificación de los residuos sólidos}

Los residuos sólidos han sido clasificados de diversas maneras, estructuralmente mantienen ciertas características desde su origen hasta su disposición final; los diferentes usos de los materiales, su biodegradabilidad, combustibilidad, reciclabilidad, etc., juegan un papel importante en la percepción de quien los clasifica (Barradas, 2009).

Así mismo, los desechos se clasifican según su fermentabilidad en orgánicos e inorgánicos; según su inflamabilidad en combustibles y no combustibles; según su procedencia en domésticos, de jardinería, de barrido, etc., y según su volumen en convencionales y especiales (OPS, 2005), adicionalmente, existen los residuos peligrosos, que debido a sus componentes (tóxico, corrosivo, reactivo, inflamable, explosivo, infeccioso, ecotóxico) pueden causar daños a la salud o al ambiente (Martínez, 2005).

\subsection{Caracterización de residuos sólidos}

El estudio de caracterización de residuos sólidos es un proceso por medio del cual se recolecta una muestra e identifica su fuente, características y cantidad de residuos generados. Esta muestra es representativa de la zona de estudio, siendo necesario implementar la fórmula del tamaño de la muestra para que los resultados reflejen reducido porcentaje de error. "La caracterización de los residuos permite planificar las acciones para el manejo de los residuos, permitiendo encontrar soluciones a los problemas que se 
presentan en las operaciones básicas de almacenamiento, recolección, transporte y disposición final, evitando el deterioro de la calidad ambiental y la salud de las personas" (Flores, 2009).

\subsection{Técnicas de tratamiento de los residuos sólidos}

"El tratamiento de los residuos sólidos es muy variado, va desde formas complicadas con el uso de alta tecnología hasta sencillas como la incineración o el entierro" (Alvarado, 2010), por su lado Mejía (2012), explica que, en la ciudad de Guatemala los sistemas de tratamiento y disposición final de los residuos sólidos son: la disposición libre o incontrolada, la disposición controlada o relleno sanitario, la disposición controlada con trituración previa, plantas de producción de compost, incineración, reciclaje y combinación de algunas de las anteriores. Las propiedades de los residuos sólidos, constituyen un elemento de significativa importancia para el uso y manejo que se haga de ellos. A partir de las características de los residuos, se toman las decisiones pertinentes relacionadas con el sistema de tratamiento más adecuado para cada caso (Alvarado, 2010).

Las nuevas tendencias en el manejo de los residuos deben resaltar en la valorización de los residuos, los programas de separación en la fuente, de reciclaje, y la sostenibilidad financiera de los servicios (OPS, 2013). Una buena gestión de los residuos persigue precisamente no perder el valor económico y la utilidad que pueden tener muchos de ellos para usarlos en vez de tirarlos (Echarri, 1998).

\section{Metodología}

La metodología que se presenta está basada en la integración de procesos metodológicos formulados por el Centro Panamericano de Ingeniería Sanitaria y Ciencias del Ambiente -CEPIS- que incluye, la determinación de la generación diaria, composición física a través del método del cuarteo y densidad de los residuos; así mismo, fue validada en campo a través experiencias aprendidas en nueve estudios de caracterización realizados en diferentes municipios del país (MARN, 2018).

\subsection{Localización del estudio}

El estudio se realizó en la plaza del mercado central del municipio de Chiquimula, departamento de Chiquimula. El área incluyó la edificación del mercado central y los alrededores hasta donde se extiende el mercado.

\subsection{Identificación de locales y puestos de piso plaza}

Por parte de la administración del mercado, se registraron hasta este año 660 puestos de venta entre locales y puestos de piso plaza.

\subsection{Determinación de número de muestras}

De acuerdo con Mejía (2017), fue necesario fijar un número mínimo de muestras tal que, los resultados a obtener reflejen cierto grado de confianza y reducido porcentaje de error. Para el cálculo del número de muestras que reflejen un alto grado de confiabilidad y reducido porcentaje de error, se utilizó la fórmula estadística siguiente:

$$
\mathrm{n}=\frac{z_{1-\propto / 2}^{2} N \sigma^{2}}{(N-1) E^{2}+Z_{1-\propto / 2}^{2} \sigma^{2}}
$$

\footnotetext{
Para el estudio se utilizaron los siguientes valores:

$\mathrm{n}=$ tamaño de la muestra

$\mathrm{N}=$ Total de locales comerciales (660).

$Z 1-\alpha=1.96$ con un grado de confianza de $95 \%$.

$\sigma 2$ = Desviación estándar de la generación de basura (0.25).

$E=$ Error permisible (0.05).
} 
Cálculo de la muestra:

$$
\mathrm{n}=\frac{(1.96)^{2}(660)(0.25)^{2}}{(660-1)(0.05)^{2}+(1.96)^{2}(0.25)^{2}}=84
$$

\subsection{Distribución de la muestra}

Conociendo el número de la muestra, se procedió a su distribución en los sectores identificados. El tamaño de la muestra se determinó en función del porcentaje de ventas con las que cuenta cada sector actualmente. En la siguiente tabla se muestran los sectores que se consideraron y la cantidad de ventas muestreados para la caracterización de residuos sólidos.

Tabla 1. Distribución de sectores del mercado central del Municipio de Chiquimula

\begin{tabular}{|l|l|r|r|r|r|}
\hline No. & Sector & $\begin{array}{r}\text { No. de } \\
\text { locales }\end{array}$ & \multicolumn{2}{|c|}{$\begin{array}{c}\text { Tamaño de } \\
\text { muestra }\end{array}$} \\
\hline 1 & Zapatería & 54 & 8.2 & 6.87 & 7 \\
\hline 2 & Verdura y fruta & 92 & 13.9 & 11.71 & 12 \\
\hline 3 & Abarrotería & 39 & 5.9 & 4.96 & 5 \\
\hline 4 & Comedor & 37 & 5.6 & 4.71 & 5 \\
\hline 5 & Carnicería & 62 & 9.4 & 7.89 & 8 \\
\hline 6 & Farmacia & 4 & 0.6 & 0.51 & 1 \\
\hline & Reparación y materiales de & & & & \\
\hline 7 & calzado & 127 & 1.2 & 1.02 & 1 \\
\hline 8 & Ropa & 119 & 18.0 & 16.15 & 15 \\
\hline 9 & Artículos varios & 98 & 14.8 & 12.47 & 12 \\
\hline 10 & Alimentos & 16 & 2.4 & 2.04 & 2 \\
\hline 11 & Refresquería & 1 & 0.2 & 0.13 & 0 \\
\hline 12 & Ferretería & 1 & 0.2 & 0.13 & 0 \\
\hline 13 & Estación de radio & 1 & 0.2 & 0.13 & 1 \\
\hline 14 & Baños & 660 & 100 & 84 & 85 \\
\hline 15 & Oratorio & & 0.13 & 0 \\
\hline Total de ventas & & & 16 \\
\hline & & & & \\
\hline
\end{tabular}

Fuente: Elaboración propia.

A pesar que 84 muestras eran las que se debía de tomar en cuenta, se decidió tomar una muestra más, dado que se consideró importante muestrear el área de los baños.

\subsection{Muestreo simple aleatorio}

La muestra se seleccionó por el método de muestreo simple aleatorio, la cual consiste en escoger de las unidades muestrales un total de locales y puestos, de tal modo que cada una tenga la misma posibilidad de ser escogida (MARN, 2018). Para ello, fue importante contar con un inventario de los sectores para que la muestra fuera representativa.

\subsection{Socialización}

La sensibilización es una etapa de suma importancia, en esta etapa se informó a los participantes el objetivo del estudio y la metodología a seguir, esta sensibilización fue en cada uno de los locales comerciales que aceptaron participar en el estudio de caracterización.

\subsection{Entrega de bolsas para recolección de} residuos sólidos

Se entregaron bolsas rotuladas con el código asignado a cada local comercial participante.

\subsection{Recolección y transporte de muestras}

Para la recolección fue importante respetar los horarios establecidos con los comerciantes. Además, el último día de recolección se indicó que la fase de campo había concluido. Este detalle fue importante para no generar molestia.

\subsection{Análisis de las muestras}

La fase de muestreo fue de ocho días sucesivos y se descartó la muestra que se tomó el primer día de la recolección. La basura recolectada del segundo al octavo día representó la generación, composición y densidad semanal de residuos sólidos mercado central del municipio de Chiquimula. 
- Medición del peso de las muestras. para el peso de las muestras en las diferentes etapas del análisis se consideró el siguiente sistema:

\section{La pesa calibrada en cero.}

2. Se colocó el recipiente vacío donde fue colocada la bolsa o los residuos y se obtuvo el peso del mismo.

3. Posteriormente se colocó la bolsa dentro del recipiente.

4. Al dato obtenido se le quitó el peso del recipiente, dando como resultado el peso real de los residuos.

- Método del cuarteo: el procedimiento empleado para establecer la composición física de los residuos sólidos se realizó aplicando el método del cuarteo de la siguiente manera:

1. Se sacó el contenido de todas las bolsas de las muestras definidas y se colocó en un área donde se mezclaron con la ayuda de palas.

2. Después de mezclar los residuos sólidos, se procedió a dividir en cuatro partes lo más equitativamente posibles.

3. Se seleccionaron dos cuartetos opuestos y se retiraron los otros dos restantes, a modo de reducir la muestra.

4. Se volvió a mezclar los residuos sólidos para realizar un nuevo cuarteo, y extraer nuevamente dos cuartetos opuestos de la muestra.

5. Se repitió el procedimiento de cuarteo hasta obtener una muestra de aproximadamente 100 libras (sin considerar el peso del tonel) que fue utilizado para realizar la composición de los residuos sólidos.

6. Al contar con la muestra se clasificó según el tipo de residuo. La composición física de los residuos sólidos del mercado se determinó por medio de la separación a mano.

7. Al clasificar según el tipo de residuo se pesó cada uno por separado, considerando que los volúmenes pequeños se debían pesar en una balanza más sensible como, por ejemplo, las que son utilizadas para pesar alimentos a granel.

8. Luego de obtener el peso de cada residuo y con base al peso inicial de la muestra de cuarteo, se sacó el porcentaje de cada residuo.

Este cálculo fue diario, al finalizar los días de estudio, se promedió los datos obtenidos para tener una muestra representativa por tipo de residuo.

Porcentaje por día $=$

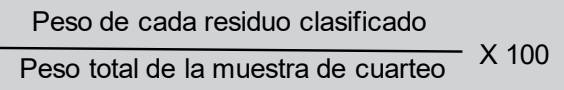

Determinación de la densidad de los residuos sólidos:

1. Se pesó el recipiente vacío y se determinó su volumen.

2. Después de mezclar los residuos previos al primer cuarteo, se llenó el tonel hasta el borde sin hacer presión dentro del mismo. Cuando el tonel estaba lleno, se golpeó el recipiente contra el suelo tres veces dejándolo caer desde una altura de $10 \mathrm{~cm}$ para que los residuos se asentaran.

3. Nuevamente se agregaron los residuos sólidos hasta llegar a la capacidad total del tonel, teniendo cuidado de no presionar al 
colocarlos; esto con el fin de no alterar el peso volumétrico que se pretendía determinar.

4. Se subió el tonel en la pesa y se anotó el peso registrado

5. El dato del peso de los residuos dividido por el volumen del tonel dio como resultado la densidad de los residuos.

$$
\text { Densidad }=\left(\frac{\text { Peso de los residuos }(\mathrm{Kg})}{\text { Volumen del tonel }\left(\mathrm{m}^{3}\right)}\right)
$$

\subsection{Análisis de los resultados}

El fin de la caracterización de los residuos sólidos generados en el mercado central del municipio de Chiquimula, fue la elaboración de alternativas a través de las cuales se les brindará un manejo adecuado a los residuos sólidos generados en la plaza del mercado central de Chiquimula.

\section{Resultados}

La caracterización de residuos sólidos en la semana de muestreo, es una estimación en cuanto a la cantidad generada en época no festiva, por lo que es importante tomar en cuenta que la generación de los residuos sólidos depende de varios aspectos, entre los cuales se puede mencionar: el incremento en los precios de los productos, las actividades en el transcurso del año, épocas festivas, cantidad de venta, temporadas de cosecha, tiempo, y clima.

\subsection{Caracterización de residuos sólidos del mercado central}

Durante la semana de muestreo se determinó el peso (lb), composición y densidad $\left(\mathrm{kg} / \mathrm{m}^{3}\right)$ de los residuos sólidos generados en la muestra proveniente de 85 locales comerciales del mercado central del municipio de Chiquimula.

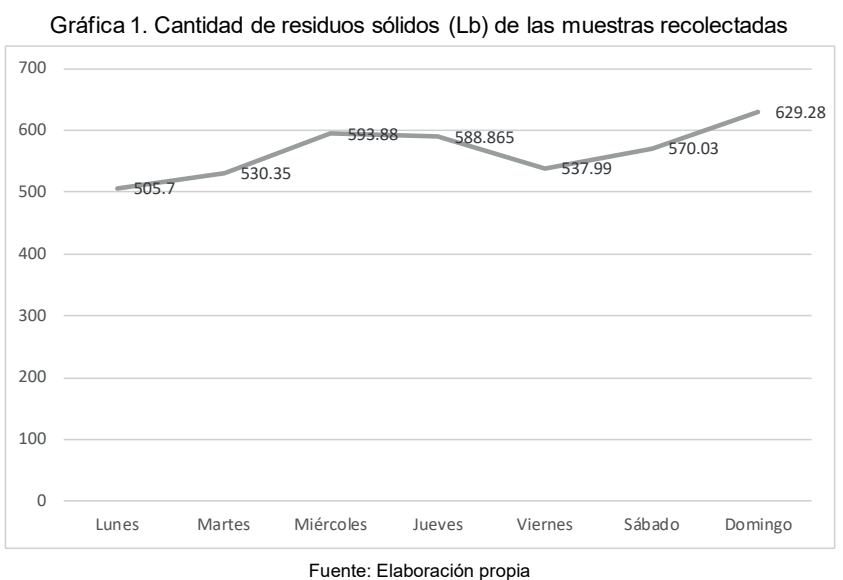

El día de menor generación de residuos sólidos en el mercado central del municipio de Chiquimula fue el día lunes con 505.7 libras, y el día de mayor generación se reportó el día domingo con un peso total de 629.28 libras. Al hacer el promedio se obtiene una generación diaria de 565.16 libras. Como se puede observar el peso total diario, durante los días de muestreo se mantiene en un rango similar. Sin embargo, es significativo considerar que la caracterización de la semana de muestreo, es una estimación en cuanto a la cantidad generada en época no festiva.

Es importante hacer la aclaración que la generación de los residuos sólidos depende de varios factores entre los que se puede mencionar: las actividades en el transcurso del año, épocas festivas, temporadas de cosecha, tiempo, clima, nivel de venta y del cambio de precios en los productos que se venden, como menciona Sepúlveda(2006), citado en López (2000:18), "un 18\% de los residuos sólidos se producen en las plazas de mercado; donde se vive una actividad comercial muy agitada y se venden diferentes productos tanto de origen orgánico como otros que están elaborados y empacados en 
materiales de plástico, vidrio, lata, etc.", por lo tanto, es importante que ha este porcentaje de generación de residuos del sector comercial se le dé una disposición final en donde existe el aprovechamiento, recuperación y reciclaje de materiales.

Tabla 2. Estimación de generación (lb) de residuos sólidos

\begin{tabular}{|l|r|r|}
\hline Día & Muestra (85 locales) & Estimación (660 locales) \\
\hline Lunes & 505.7 & 3926.611765 \\
\hline Martes & 530.35 & 4118.011765 \\
\hline Miércoles & 593.88 & 4611.303529 \\
\hline Jueves & 588.865 & 4572.363529 \\
\hline Viernes & 537.99 & 4177.334118 \\
\hline Sábado & 570.03 & 4426.115294 \\
\hline Domingo & 629.28 & 4886.174118 \\
\hline Generación semanal & 3956.095 & 30717.91412 \\
\hline \multicolumn{3}{|r|}{ Fuente: Elaboración propia. }
\end{tabular}

Durante la semana de estudio se obtuvo una producción semanal de residuos de 3,956.095 libras, haciendo la proyección correspondiente al total de locales (660) del mercado central se puede concluir que la estimación de residuos sólidos es de aproximadamente 30,717.91 libras semanal.

De acuerdo con MARN (2018), en Guatemala se ha presentado un desarrollo en las últimas décadas que se evidencia por medio del crecimiento económico, expansión urbanística y el incremento en el sector industrial; estos van directamente asociados con la demanda de recursos y servicios para satisfacer las necesidades de la población, que reflejan un patrón de consumo que sobrepasa la capacidad del sistema, produciendo un aumento en la generación de residuos y desechos sólidos.

En definitiva, los residuos no recolectados y mal manejados en el mercado central de Chiquimula, constituyen uno de los grandes factores que ejercen presión al ambiente, tomando en consideración esto es importante la búsqueda de alternativas para aprovechar el alto potencial que tienen y que a su vez ayude a reducir el problema que producen.

Gráfica 2. Composición física de residuos sólidos

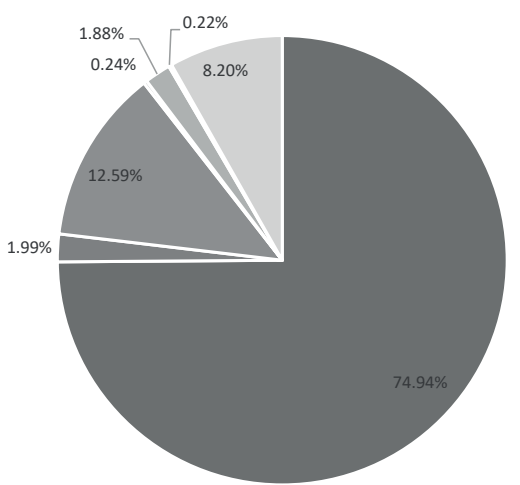

- Materia orgánica - Papel higiénico - Plástico $=$ Metal - Vidrio =Otros = Papel y cartón

Fuente: Elaboración propia.

Por la composición de los residuos generados en el mercado, los residuos orgánicos son los que presentan mayor porcentaje con el $74.94 \%$ del total de residuos, estos están compuestos básicamente por residuos de comida proveniente de los comedores, restos de frutas y verduras (principalmente tomate, zanahoria, rábano, papa, aguacate, hojas, hierbas, etc.); en menor cantidad huesos por parte de las carnicerías del mercado, en este punto es importante resaltar que únicamente se consideró los residuos que llegan al botadero, puesto que varios vendedores venden parte de los desperdicios para alimento de animales.

Seguidamente se encuentra el plástico con el $12.58 \%$ (todo tipo de plástico), papel y cartón con el $8.19 \%$, proveniente principalmente de las abarroterías y zapaterías, papel higiénico con el $1.99 \%$, vidrio con el $1.88 \%$, metal con el $0.24 \%$ y la clasificación de otros con el $0.22 \%$ del peso total, que incluye pedazos de telas y materiales de reparación de calzado. 
Según los datos anteriores, existe un alto potencial de los residuos sólidos generados en el mercado central, en virtud que, en su mayoría se pueden utilizar en métodos de aprovechamiento como la producción de compostaje y el reciclaje en el caso de residuos recuperables como el plástico, papel y cartón principalmente.

La composición física de los residuos a nivel nacional está caracterizada por su alto porcentaje de materia orgánica, como lo establece el MARN (2018), el 53\% del total de residuos en el país son de origen orgánico. Sin duda alguna, las plazas de mercado presentan un carácter más definido aún en cuanto a la composición de los residuos sólidos, dado que allí se concentran los expendios de verduras, frutas, carnes, abarrotes y otros, así mismo indica Benavente (2012), en su estudio de caracterización en el mercado de San Juan Comalapa los residuos orgánicos representan el $69.49 \%$ del total de residuos.

Si bien es cierto los residuos orgánicos representan el más alto porcentaje en este estudio pudiéndose aprovechar para la producción de compostaje, así mismo los residuos inorgánicos recuperables, pueden ser recolectados, clasificados y vendidos a empresas dentro del Departamento de Chiquimula, que se dedican a la compra de materiales reciclables.

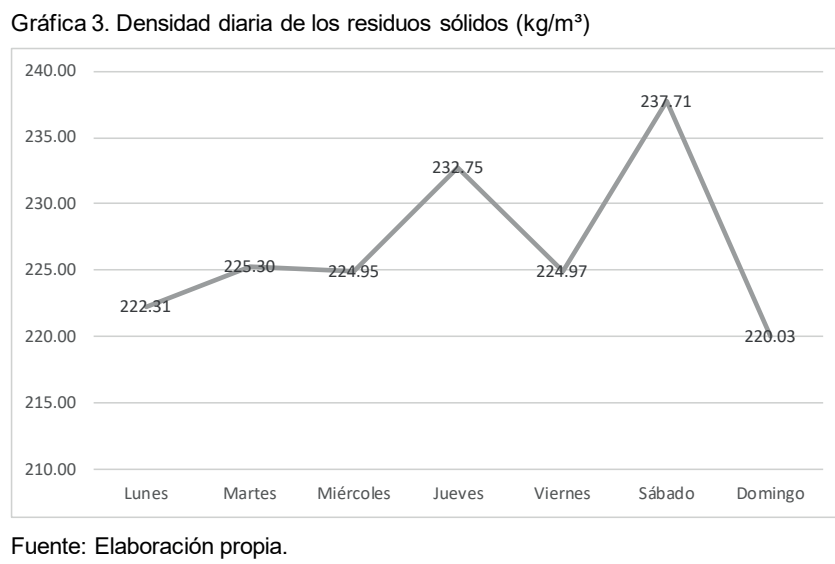

La densidad de los residuos sólidos se mantuvo durante los días de muestreo en valores similares encontrándose densidades que van desde los $220.03 \mathrm{~kg} / \mathrm{m}^{3} \mathrm{a} 237.71 \mathrm{~kg} / \mathrm{m}^{3}$. El cambio en el valor de la densidad los días jueves y sábado que muestran los valores más altos, se debe a la menor cantidad de volumen medido en las muestras recolectadas en relación al peso de los mismos, se puede inferir que durante estos días los residuos sólidos son de mayor peso específico por unidad de volumen. A diferencia de lo que sucede el día domingo que fue el día de mayor recaudación de residuos tanto en peso con volumen; con un peso de $286.03 \mathrm{~kg}$ sobre un volumen de $1.3 \mathrm{~m}^{3}$; lo que dio como resultado la menor densidad de todos los días muestreados.

\subsection{Alternativas para el manejo de los resi- duos sólidos}

Con el manejo adecuado de los residuos sólidos que se producen en el mercado central de Chiquimula, se pueden generar actividades económicas conexas tales como la producción de compost (abono orgánico) y el reciclaje de productos (papel, cartón, vidrio, metal y plástico), los cuales tienen canales de comercialización y mercados establecidos. Por lo tanto, las alternativas propuestas están enfocada principalmente en: 
- Separación y recolección diferenciada de residuos

- Elaboración de compost

- Comercialización y Sensibilización y educación ambiental

\subsubsection{Separación y recolección diferenciada}

La separación es una operación necesaria en la recuperación de materiales reutilizables y reciclables. Una manera factible es conseguir la separación en el punto de generación. Se requiere que la Municipalidad de Chiquimula establezca la recolección separada de los residuos en por lo menos tres categorías (orgánico, inorgánico comercializable e inorgánico no comercializable):

- Orgánico: todo residuo de origen natural que se descompone y que puede servir para la producción de compost.

- Inorgánico con valor: corresponde a todo tipo de residuo que se pueda vender.

- Inorgánico sin valor (no reciclable): son todos los residuos que no contribuyen a producir nuevos elementos y por consiguiente no tienen valor o cuyo costo de recuperación es tan alto que no es económicamente viable, y que, por lo tanto, serán trasladados al vertedero municipal para su disposición final, el cual está ubicado en la parte posterior de la colonia Altamira jurisdicción de la aldea Petapilla en las afueras de la ciudad de Chiquimula, este lugar fue seleccionado por las autoridades municipales para evitar principalmente la contaminación visual y es el autorizado desde años atrás por la autoridad municipal de Chiquimula para la disposición final de los residuos del municipio.
A partir de la separación se puede implementar el proceso de reciclaje para la transformación de los residuos sólidos orgánicos nuevamente en materia prima (abono orgánico); y para los residuos sólidos inorgánicos reciclables se puede buscar como alternativa empresas intermediarias que compren dichos materiales con valor comercial dentro del Municipio de Chiquimula o con empresas recicladoras a nivel nacional.

\subsubsection{Elaboración de compost}

Tomando en cuenta que los residuos sólidos orgánicos que se producen en el mercado central constituyen el $74.94 \%$ del total de residuos, es indispensable buscar una alternativa que contribuya al manejo adecuado, potenciando los productos finales y minimizando los impactos ambientales que este conlleva. Una de las técnicas más usadas para el aprovechamiento de los residuos sólidos orgánicos es el compostaje; el cual se define como la descomposición de residuos orgánicos por la acción microbiana, cambiando la estructura molecular de los mismos (Jaramillo y Zapata, 2008), por lo que dentro de esta propuesta se plantea la elaboración de compost a través de la técnica de compostaje de hileras volteadas tradicionales.

El tipo de operación que se emplea en cada planta de compostaje, dependerá del financiamiento, recursos humanos y técnicos disponibles, considerando que la Municipalidad de Chiquimula carece de recursos como lo afirma Ayala (2010), la municipalidad de Chiquimula tiene insuficiente capacidad para el cumplimiento adecuado de funciones específicas de la basura, por lo que se recomienda la "planta de operación semi-industrial", este tipo de planta se diseña con operación manual en su mayoría y con alguna maquinaria necesaria para el proceso de 
compostaje entre los cuales se incluye:

- Maquinaria procesadora de material como trituradoras, tamizadoras y mezcladoras

- Vehículos: utilizados para cualquiera de las actividades en que se necesite transporte dentro del proceso

- Herramientas manuales: como palas, rastrillos, azadones, picos, carretillas, regaderas, mangueras, etc.

- Herramientas técnicas: generalmente utilizadas para el control técnico del proceso, tales como: termómetros, higrómetros, medidores de $\mathrm{pH}$, etc. Estos son equipos básicos que ayudan a controlar y monitorear las condiciones del proceso.

La técnica de compostaje que se propone es el de hileras volteadas tradicionales, en esta técnica los residuos sólidos orgánicos se agrupan en filas o hileras casi triangulares sobre superficies sólidas, que son volteadas en repetidas ocasiones a lo largo del proceso para oxigenar todo el material. Se recomienda una altura de entre 1.2 a $1.8 \mathrm{~m}$ y un ancho de 2.4 a 3.6 metros. La longitud de la pila solo queda limitada por las dimensiones o la distribución de la planta, ya que esta dimensión no está restringida por el proceso (Barrena, 2006). El desarrollo total se suele lograr en más de tres semanas, después se deja el compost curándose durante 12 semanas (Barros, 2010).

\subsubsection{Comercialización de productos recicla- bles}

Por lo general los residuos tienen en su composición una adecuada cantidad de materiales que pueden reciclarse. De una manera práctica, los componentes recuperables son aquellos para los cuales existe un mercado, tal que justifican su separación. Los materiales recuperables de los residuos sólidos del mercado central en este estudio incluyen principalmente: plástico (todo tipo de plástico) el $12.59 \%$, papel y cartón con el $8.20 \%$.

En el caso del plástico existen empresas que se dedican al reciclaje. Entre estas, el plástico más reciclado en Guatemala es el polietileno tereftalato (más conocido por sus siglas en inglés PET, polyethylene terephthalate), que es un tipo de plástico muy usado en envases de bebidas y textiles; a pesar que en este estudio el plástico representa el $12.59 \%$, no todo el plástico que se produce en el mercado central reúne las condiciones necesarias para su reciclaje, por lo tanto, para fines de esta propuesta se recomienda solo tomar en cuenta el plástico PET, por ser el más reciclado ya que es flexible, no se quiebra, no corta y es más económico comparado con otros. Su tecnología de reciclabilidad es conocida y hay capacidad instalada para hacerlo, además es más fácil de identificar, comercializar y además hay mucho volumen.

En el municipio de Chiquimula se encuentra la empresa recicladora de oriente, que es una empresa intermediaria que se dedica a la recolección de basura clasificada en un centro de acopio ubicado en el kilómetro 167 , la cual vende los residuos a empresas recicladoras del país que se dedican a la trasformación de residuos recuperados y se devuelve a los materiales su potencial de reincorporación como materiales para la fabricación de nuevos productos.

La empresa recicladora de oriente, en el caso del plástico únicamente compra el plástico polietileno tereftalato -PET-, el cual se identifica por el símbolo de reciclaje con el número 
uno, pagando a $Q 0.60$ centavos la libra el plástico transparente, así mismo compra el plástico PET de color celeste y gris pagando Q 0.30 centavos por libra. Ambos plásticos son PET (transparente, celeste y gris), las cuales incluyen un símbolo con el número uno, dentro de las siete categorías de plástico identificados, la única característica que los diferencia es el color, sin embargo, su precio varía porque el plástico transparente tiene un mayor valor en el mercado.

En el caso del papel y cartón deben de entregarse limpio, seco, libre de grasa, de humedad y separado según el tipo. En el caso del papel y cartón la empresa recicladora de oriente compra el cartón a Q 0.05 centavos la libra, y el papel a $Q 0.35$ centavos la libra.

\subsubsection{Sensibilización y educación ambiental} Como complemento indispensable se debe de planificar estrategias educativas dirigidas a la educación sanitaria y promoción de la salud a los inquilinos del mercado central, lo que influirá en la manipulación de los residuos, facilitando la separación, recolección, almacenamiento, tratamiento y disposición final de manera eficiente, económica y ambientalmente segura.

\section{Conclusiones}

De las muestras provenientes de los 85 locales comerciales del mercado central se obtuvo una generación de 3,956.095 libras; por lo que se pude estimar que en el mercado central del municipio de Chiquimula se generan aproximadamente 30,717.91 libras de residuos sólidos semanales; sin embargo, es importante considerar que la generación de residuos está relacionada con varios factores por lo que su generación podrá variar en el transcurso del año.
Mediante este estudio, se logró determinar la composición de los residuos generados en el mercado central del municipio de Chiquimula, siendo predominante el componente orgánico con el $74.94 \%$ seguidamente se encuentra el plástico con el $12.59 \%$, papel y cartón con el $8.20 \%$, papel higiénico con el $1.99 \%$, vidrio $1.88 \%$, metal con el $0.24 \%$ y la clasificación de otros (tela y materiales de reparación de calzado) con el $0.22 \%$ del peso total de los residuos sólidos.

La densidad de los residuos sólidos se mantuvo durante los días de muestreo en valores similares encontrándose densidades que van desde los $220.03 \mathrm{~kg} / \mathrm{m}^{3}$ a $237.71 \mathrm{~kg} / \mathrm{m}^{3}$.

De acuerdo con la caracterización física de los residuos sólidos del mercado central del municipio de Chiquimula el manejo de los residuos sólidos debe de estar principalmente enfocado en el tratamiento de los residuos orgánicos ya que representa el mayor porcentaje de residuos generados; y al reciclaje de productos inorgánicos reciclables como plástico (PET), papel y cartón; considerando que son los residuos que tienen mayor potencial económico para su comercialización.

Se identificaron alternativas a través de las cuales se pretende manejar y disponer los residuos sólidos generados en la plaza del mercado central del municipio de Chiquimula, que contribuyan a la disminución de la contaminación y a la generación de ingresos económicos, entre las cuales se incluye: la separación y recolección diferenciada de residuos, elaboración de compost, comercialización de productos reciclables y sensibilización. 


\section{Referencias bibliográficas}

Aguilar, G. y A. Iza (2009). Derecho Ambiental en Centroamérica. Tomo II, (Suiza): UICN, editorial

Alvarado, E. (2010). Evaluación y propuesta de mejora de la situación actual del manejo de los desechos sólidos en el municipio de Palencia, departamento de Guatemala, (Guatemala): Universidad de San Carlos de Guatemala.

Ayala, G. (2010). Manejo municipal y caracterización de los desechos sólidos de la ciudad de Chiquimula, departamento de Chiquimula, (Chiquimula): Universidad de San Carlos de Guatemala.

Barradas, A. (2009). Gestión Integral de Residuos Sólidos Municipales, (México): Instituto Tecnológico de Minatitlán.

Barrena, R. (2006). Compostaje de residuos sólidos orgánicos. Aplicacion de tecnicas respirometricas en el seguimiento del proceso, (Barcelona): Universidad Autonoma de Barcelona.

Barros, D. (2010). Evaluación de rendimientos en el sistema de gestión final de los residuos sólidos del Cantón Mejía, (Quito): Escuela Politecnica Nacional.

Benavente, L. (2012). Propuesta técnica para el manejo de desechos sólidos generados en el sector del mercado del municipio de San Juan Comalapa, Chimaltenango, (Guatemala): Universidad Rafael Landivar.

Contreras, M. (2008). Evaluación de expriencias locales urbanas desde el concepto de sostenibilidad: el caso de los desechos sólidos del municipio de Los Patios (Norte de Santander, Colombia), (Colombia): Universidad Nacional de Colombia.
Echarri, L. (1998). Ciencias de la tierra y del medio ambiente, (España). Universidad de Navarra.

Férnandez, A. y M. Sánchez (2007). Guía para la gestión integral de los desechos sólidos urbanos, (Cuba): Organización de las Naciones Unidas para el Desarrollo Industrial.

Flores, J. (2009). Implementación del sistema de manejo integral de residuos sólidos urbanos en el distrito de Las Lomas. Estudio de caracterización de residuos sólidos, (Perú): Municipalidad Distrital de Las Lomas.

Jaramillo, G. y L. Zapata (2008). Aprovechamiento de los residuos sólidos orgánicos en Colombia, (Colombia): Universidad de Antioquia.

MARN (Ministerio de Ambiente y Recursos Naturales) (2018). Guía para elaborar estudios de caracterización de residuos sólidos comunes, (Guatemala): Departamento para el manejo de los residuos y los desechos sólidos.

MARN (Ministerio de Ambiente y Recursos Naturales) (2018). Guía para la identificación gráfica de los residuos sólidos comunes, (Guatemala): Departamento para el manejo de residuos y desechos sólidos.

Martínez, J. (2005). Guía para la gestión integral de residuos peligrosos. Fundamentos tomo I, (Uruguay): Centro Coordinador del Convenio de Basilea para America Latina y el Caribe.

Mejía, A. (2017). Plan de manejo integral de los residuos sólidos orgánicos en el mercado la Parroquia, ciudad de Guatemala, (Guatemala): Universidad Rafael Landivar. 
Mejía, J. (2012). Propuesta de manejo de residuos sólidos aplicable a pequeñas y medianas empresas productoras de plásticos de la ciudad de Guatemala, fundamentada en el acuerdo municipal 028-2002, (Guatemala): Universidad Rafael Landivar.

OPS (Organización Panamericana de la Salud) (2013). Salud, ambiente y desarrollo sostenible: hacia el futuro que queremos, (Washington, D.C): Programa Especial de Desarrollo Sostenible y Equidad en Salud.

OPS (Organización Panamericana de la Salud) (2005). Informe de la evaluación regional de los servicios de manejo de residuos sólidos municipales en America Latina y el Caribe, (Washington, D.C): s.n.
SEDESOL (Secretaría de Bienestar Social) (2005). Manual técnico sobre generación, recolección y transferencia de residuos sólidos municipales, (México): s.n.

URL (Universidad Rafael Landivar). (2009). Gestión ambiental y gobernabilidad local, (Guatemala): Instituto de Agriculltura, Recursos Naturales y Ambiente.

Zamora, J. (2013). Plan de manejo ambiental de desechos sólidos del municipio de San Andrés Itzapa, Chimaltenango, (Guatemala): Universidad de San Carlos de Guatemala.

\section{Sobre autor}

\section{Andrea Karina Casasola Garza}

Ingeniera en Gestión Ambiental Local, Profesional de apoyo para el desarrollo de la mujer, juventud y niñez en sede departamental, Ministerio de Agricultura, Ganadería y Alimentación -MAGA-

Copyright (c) 2021 Andrea Karina Casasola Garza

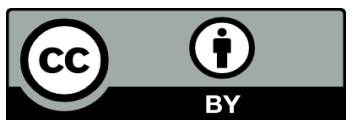

Este texto está protegido por una licencia Creative Commons $\underline{4.0}$

Usted es libre para Compartir — copiar y redistribuir el material en cualquier medio o formato- y Adaptar el documento — remezclar, transformar y crear a partir del material- para cualquier propósito, incluso para fines comerciales, siempre que cumpla la condición de:

Atribución: Usted debe dar crédito a la obra original de manera adecuada, proporcionar un enlace a la licencia, e indicar si se han realizado cambios. Puede hacerlo en cualquier forma razonable, pero no de forma tal que sugiera que tiene el apoyo del licenciante o lo recibe por el uso que hace de la obra.

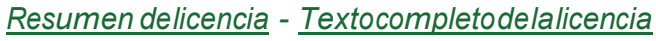

Revista digital: ISSN 2707-9643

Revista impresa: ISSN 2313-786X

Editorial Servi Prensa, Guatemala

https://doi.org/10.37533/cunsurori.v8i1.66
Vol. 8 No. 1 Enero - Diciembre 2021 
